\title{
Blood Pressure Variability and Prognosis in Hemodialysis Patients: A Systematic Review and Meta-Analysis
}

\author{
Yuliang Zhao a, b Letian Yang ${ }^{a, b}$ Shaobin Yua, ${ }^{a}$ Stephen Salerno ${ }^{c}$ Yi Li $^{c, d}$ \\ Tianlei Cui ${ }^{a, b}$ Ling Zhang ${ }^{a, b}$ Ping Fu ${ }^{a, b}$ \\ aDivision of Nephrology, West China Hospital of Sichuan University, Chengdu, China; ${ }^{\text {b} K i d n e y ~ R e s e a r c h ~ I n s t i t u t e, ~}$ \\ West China Hospital of Sichuan University, Chengdu, China; 'Department of Biostatistics, School of Public Health, \\ University of Michigan, Ann Arbor, MI, USA; ${ }^{~}$ Kidney Epidemiology and Cost Center, University of Michigan, \\ Ann Arbor, MI, USA
}

\section{Keywords}

Blood pressure variability · Hemodialysis · Prognosis ·

Cardiovascular $\cdot$ Mortality

\begin{abstract}
Background: The prognostic value of blood pressure variability (BPV) in patients receiving hemodialysis is inconclusive. In this study, we aimed to assess the association between BPV and clinical outcomes in the hemodialysis population. Methods: Pubmed/Medline, EMBASE, Ovid, the Cochrane Library, and the Web of Science databases were searched for relevant articles published until April 1, 2020. Studies on the association between BPV and prognosis in patients receiving hemodialysis were included. Results: $A$ total of 14 studies (37,976 patients) were included in the analysis. In patients receiving hemodialysis, systolic BPV was associated with higher all-cause (hazard ratio [HR]: 1.13; 95\% confidence interval [Cl]: 1.07-1.19; $p<0.001)$ and cardiovascular (HR: 1.16; 95\% Cl: 1.10-1.22; $p<0.001$ ) mortality. In the stratified analysis of systolic BPV, interdialytic systolic BPV, rather than 44-h ambulatory systolic BPV or intradialytic systolic BPV, was identified to be related to both allcause (HR: $1.11 ; 95 \% \mathrm{Cl}: 1.05-1.17 ; p=0.001)$ and cardiovascular (HR: 1.14; 95\% Cl: 1.06-1.22; $p<0.001$ ) mortality.
\end{abstract}

Among the different BPV metrics, the coefficient of variation of systolic blood pressure was a predictor of both all-cause $(p=0.01)$ and cardiovascular $(p=0.002)$ mortality. Although diastolic BPV was associated with all-cause mortality (HR: $1.09 ; 95 \% \mathrm{Cl}: 1.01-1.17 ; p=0.02$ ) in patients receiving hemodialysis, it failed to predict cardiovascular mortality (HR: 0.86 ; $95 \% \mathrm{Cl}: 0.52-1.42 ; p=0.56)$. Conclusions: This metaanalysis revealed that, in patients receiving hemodialysis, interdialytic systolic BPV was associated with both increased all-cause and cardiovascular mortality. Furthermore, the coefficient of variation of systolic blood pressure was identified as a potentially promising metric of BPV in predicting all-cause and cardiovascular mortality. The use of 44-h ambulatory systolic BPV, intradialytic systolic BPV, and metrics of diastolic BPV in the prognosis of the hemodialysis population require further investigation (PROSPERO registry number: CRD42019139215).

(c) 2020 The Author(s)

Published by S. Karger AG, Basel

\section{Introduction}

Blood pressure is a leading risk factor for cardiovascular disease (CVD), but blood pressure variability (BPV) has historically been viewed as merely a phenomenon to karger@karger.com www.karger.com/kdd

Karger $\frac{1}{\%}$

GOPEN ACCESS
(C) 2020 The Author(s)

Published by S. Karger AG, Basel

This article is licensed under the Creative Commons AttributionNonCommercial-NoDerivatives 4.0 International License (CC BYNC-ND) (http://www.karger.com/Services/OpenAccessLicense) Usage and distribution for commercial purposes as well as any distribution of modified material requires written permission.
Yuliang Zhao

Division of Nephrology, West China Hospital of Sichuan University

37\# Guoxue Alley, Wuhou Distr.

Chengdu 610041 (China)

dr.zhaoyuliang@gmail.com 
be overcome for precise blood pressure measurement. Recent studies have indicated the potential of BPV in predicting target organ damage and cardiovascular events. Several meta-analyses have confirmed the association between increased BPV and cardiovascular mortality in the general hypertensive population [1-4]. Hypertension is also common in patients with $\mathrm{CKD}$, with the prevalence in patients receiving hemodialysis ranging from 50 to $90 \%$ [5]. Severe blood volume fluctuations during dialysis sessions and interdialytic periods may result in higher BPV in patients undergoing hemodialysis than in patients with simple hypertension $[6,7]$. However, the use of $\mathrm{BPV}$ in assessing the prognosis of individuals receiving dialysis is a matter of contention. In 1999, Tozawa et al. [8] reported that the coefficient of variation (CV) of systolic blood pressure, an indicator of BPV, maybe a significant prognostic factor for all-cause mortality in patients with ESRD. In 2013, Di Iorio et al. [9], in a study involving 1,088 patients, suggested the predictive value of BPV in cardiovascular outcomes. On the contrary, in 2018, Sarafidis et al. [10] pointed out that increased BPV was not related to prevalent CVDs, even after adjusting for pulse wave velocity and other risk factors. A fully adjusted secondary analysis of the HEMO study failed to recognize a statistically significant association between pre-dialytic systolic BPV and all-cause mortality [11]. Our understanding of BPV and hemodialysis has also been compromised because of statistical and methodological heterogeneity in the available publications. For example, crude unadjusted models could have over-estimated the predictive value of BPV as compared to multivariable Cox regression models. Studies also vary in the use of different BPV metrics, measurement protocols, and cohort characteristics. Because results from the current literature are inconclusive, we performed a systematic review and meta-analysis to assess whether increased $\mathrm{BPV}$ is associated with poor prognosis in patients receiving hemodialysis.

\section{Materials and Methods}

\section{Data Sources and Searches}

PubMed/Medline, EMBASE, Ovid, the Cochrane Library, and the Web of Science databases were searched for relevant publications, with the last literature search being performed on April 1, 2020. The free word search terms were as follows: "blood pressure," "variability," and "renal or kidney" or "dialysis or hemodialysis" or "renal replacement therapy." The MESH terms "Blood Pressure" (MESH), "Kidney Failure, Chronic" (MESH), "Renal Dialysis" (MESH), and "Renal Replacement Therapy" (MESH) were also used to facilitate the literature search. The PubMed/
Medline search strategy is described in online suppl. Table 1 (see www.karger.com/doi/10.1159/000511295 for all online suppl. material), which was adapted for the other databases accordingly. We imposed no date restrictions. Reference lists of the identified articles were also searched manually for relevant studies.

\section{Study Selection}

All the identified articles were screened by 2 reviewers (Z.Y. and Y.L.) independently, with adjudication by a third reviewer (Y.S.). The inclusion criteria were as follows: (a) patients receiving hemodialysis aged $>18$ years; (b) cohort study design with followup; (c) analysis of the association between BPV and mortality or cardiovascular outcomes; and (d) sufficient data present in the hazard ratios (HRs) and 95\% confidence intervals (CIs). Exclusion criteria were as follows: (a) assessment of only intermediate outcomes, such as left ventricular hypertrophy; (b) BPV as an end point; and (c) non-English articles. For multiple articles from the same research, we included the study with the most information.

\section{Data Extraction and Quality Assessment}

Using prespecified standardized forms, 2 independent reviewers (Z.Y. and Y.L.) examined each of the included studies. They extracted data on study characteristics, quality indices (using the Quality in Prognosis Studies [QUIPS] tool [12-14]), BPV measurement, and research outcomes. Discrepancies were resolved by discussion with a third investigator (Y.S.). For those studies conducting more than one multivariate analysis, results from the fully adjusted models were considered. If outcomes from multiple BPV metrics were available in a single study, we chose the BPV metric with most information for the general analysis. Similarly, results derived from systolic BPV in the form of a continuous variable were preferred to results from diastolic BPV as a categorical variable. We combined the HRs for study subgroups before inclusion if no overall data were available. When relevant data were not described, we contacted the corresponding authors for details. Any data that were not reported in the full text (or online suppl. material) or not provided by the author were described as non-reported.

\section{Evaluation of Study Quality}

The QUIPS tool is a well-accepted approach to assess the risk of bias in studies of prognostic factors [12]. It has been used or adapted by different kidney disease research teams conducting systematic reviews addressing prognosis $[13,14]$. In this analysis, we assessed the risk of bias using the QUIPS tool. Two researchers (Y.L. and Y.S.) independently extracted data in the following 6 domains: study participation, study attrition, prognostic factor measurement, outcome measurement, study confounding, and statistical analysis and reporting. Any disagreements were resolved through group discussions (Y.L., Y.S., and Z.Y.).

\section{Data Synthesis and Analysis}

The primary outcomes were all-cause and cardiovascular mortality. The secondary outcome was a composite of cardiovascular events, including both cardiovascular deaths and nonfatal cardiovascular events. Considering the inherent heterogeneity between these studies, we assumed the presence of statistical heterogeneity. In the meta-analysis, the pooled effects were expressed as standardized HRs and 95\% CIs under a random-effects model (I-V heterogeneity). A $p$ value of $<0.05$ was considered statistically significant. Heterogeneity was assessed using the $\chi^{2}$ test and $I^{2}$ statis- 


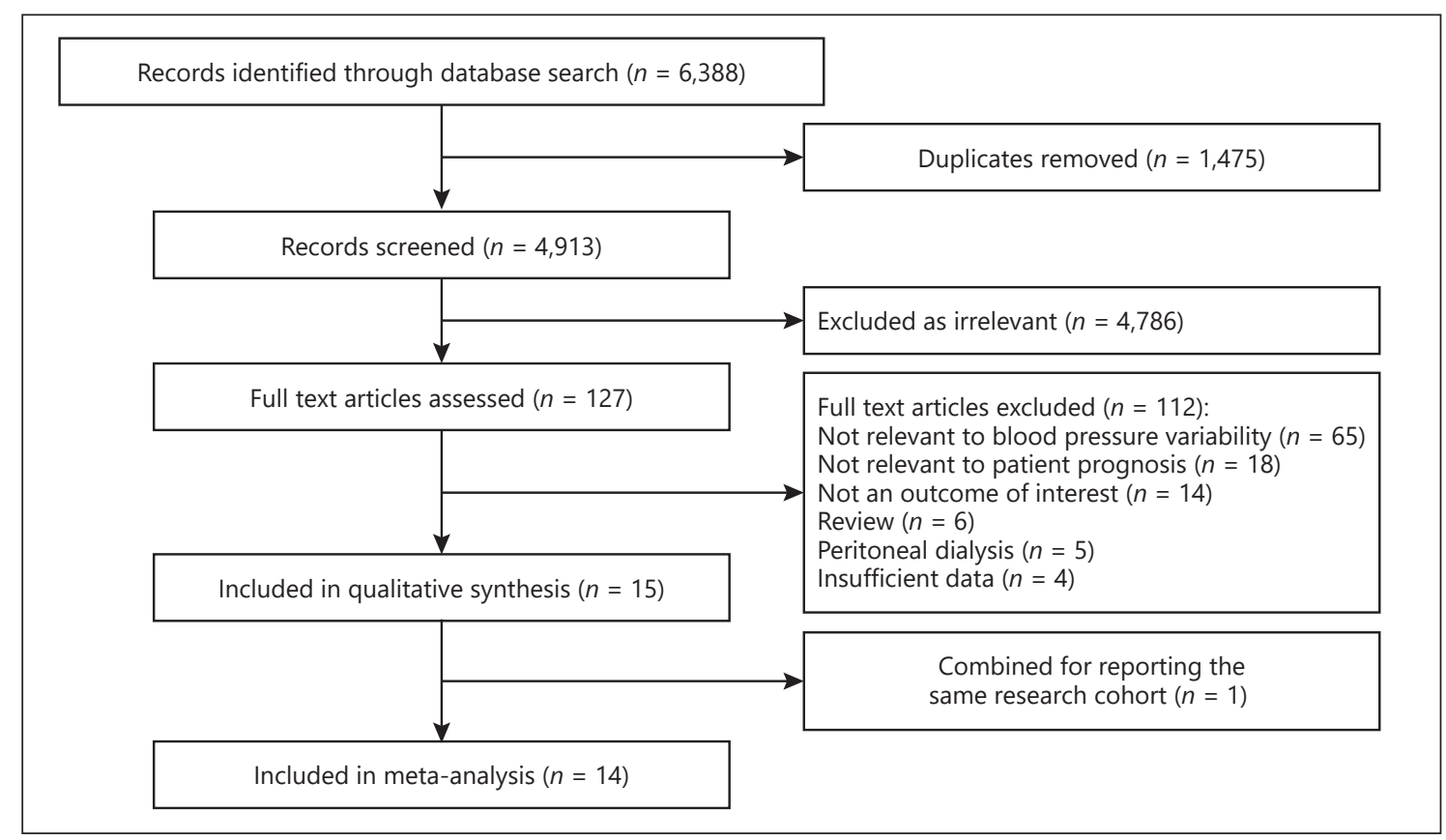

Fig. 1. PRISMA flow chart of study selection. BPV, blood pressure variability.

tics. Meta-regression analyses (restricted maximum likelihood method) were used to assess the sources of heterogeneity. Subsequently, we performed additional subgroup analyses based on the type of BPV, BPV metrics, and study characteristics considered to be clinically important or suggested by meta-regression as contributors to heterogeneity. Publication bias was analyzed using the statistical assessment of the Egger's test. In case of significant publication bias, a nonparametric trim and fill method was used to verify the stability of the result. Sensitivity analysis was conducted by sequentially deleting a single study each time to identify the potential influence of the individual study. Data analyses were performed using STATA 14.0 (STATA Corp., College Station, TX, USA) by 2 different reviewers (Z.Y. and S.S.) independently, with adjudication by a third reviewer (L.Y.).

\section{Results}

The initial search yielded 4,913 articles. After screening the titles and abstracts, we excluded 4,786 irrelevant articles. The remaining 127 potentially relevant articles were subjected to full-text assessment. On the basis of our study criteria, 113 articles were further excluded. Thus, a total of 14 studies were included in this systematic review and meta-analysis (Fig. 1) [8-11, 15-24].

\section{Study Characteristics}

The 14 eligible studies were published from 1999 to 2019 , with a total of 37,976 patients. The follow-up dura- tion ranged from 185 days to 14 years. There were 7 studies from Western countries (USA, UK, Italy, and Greece) and 7 from Asian countries (Japan, China, and South Korea). All-cause mortality was reported by 12 studies and cardiovascular mortality by 10 studies. Eight of the 14 studies reported both all-cause and cardiovascular mortality (Table 1).

\section{Quality Assessment}

The QUIPS tool was used to assess the quality of the included studies. Most studies were rated as low risk in the domains of study participation $(11 / 14,78.57 \%)$, outcome measurement $(12 / 14,85.71 \%)$, prognostic factor measurement $(13 / 14,92.86 \%)$, and study confounding $(14 / 14,100 \%)$, whereas $64.29 \%(9 / 14)$ and $64.29 \%(9 / 14)$ of the included studies were rated as the moderate risk in the domains of study attrition and statistical analysis/reporting, respectively. None of the studies were identified as high risk in any of these domains (online suppl. Table 2).

\section{BPV Measurement}

Three studies measured intradialytic BPV during dialysis, and 3 studies analyzed 44-h ambulatory BPV during dialysis intervals. Most of the included studies focused on interdialytic (dialysis to dialysis) or intervisit (visit to visit) BPV. All the studies reported systolic BPV, 


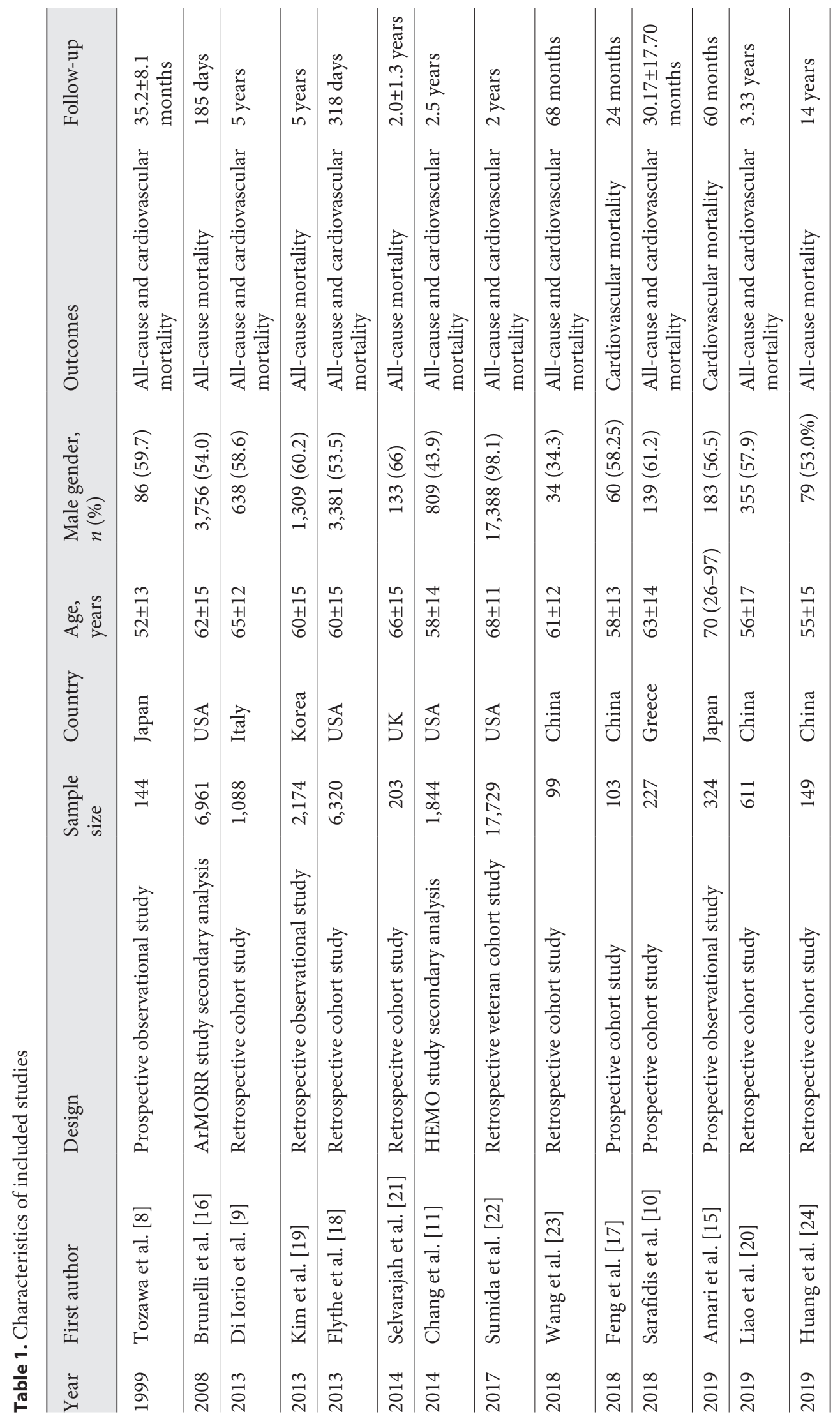


Table 2. BPV measurement methodology

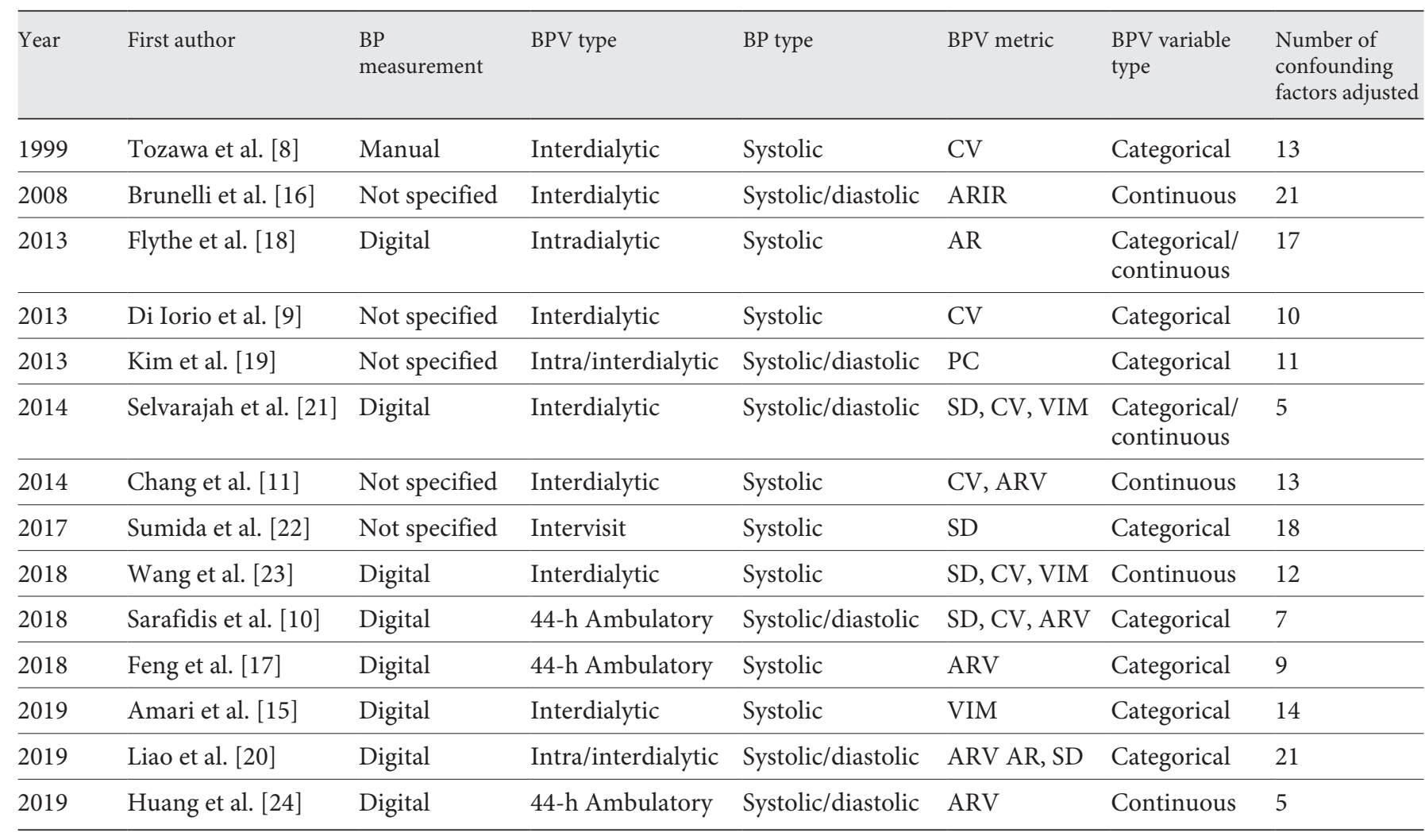

AR, absolute residual; ARIR, average residual-intercept ratio; ARV, average real variability; BP, blood pressure; BPV, blood pressure variability; $\mathrm{CV}$, coefficient of variation; $\mathrm{PC}$, pressure change; $\mathrm{SD}$, standard deviation; VIM, variation independent of mean.

and 6 reported diastolic BPV as well. To quantify BPV, various metrics were used: standard deviation $(\mathrm{SD}), \mathrm{CV}$, variation independent of the mean (VIM), average real variability (ARV), absolute residual (AR), average residual-intercept ratio (ARIR), and pressure change (PC; the difference between the highest and lowest $\mathrm{BP}$ readings). $\mathrm{CV}$ and SD were the most widely used BPV metrics. BPV was converted into a categorical variable in 10 studies and into a continuous variable in 6 studies (Table 2).

\section{All-Cause Mortality}

Systolic BPV

Twelve studies, involving 37,549 patients, examined the prognostic significance of systolic BPV for all-cause mortality. The overall analysis indicated that increased systolic BPV was associated with higher risk of all-cause mortality in patients receiving hemodialysis (HR: 1.13; 95\% CI: 1.07-1.19; $p<0.001$ ) (Fig. 2a). Interdialytic systolic BPV was found to be related to all-cause mortality
(HR: $1.11 ; 95 \%$ CI: $1.05-1.17 ; p=0.001$ ), but neither $44-\mathrm{h}$ ambulatory systolic BPV (HR: 1.25; 95\% CI: 0.99-1.57; $p=0.058$ ) nor intradialytic systolic BPV (HR: 1.08; 95\% CI: $0.93-1.26 ; p=0.301$ ) was identified to have a similar effect (Fig. 2b).

We examined the possible sources of substantial underlying heterogeneity $\left(I^{2}=51.6 \%, p=0.019\right)$ using metaregression analysis (online suppl. Table 3). The results, although statistically insignificant, suggested that heterogeneity may in part be explained by the region of study origin $(p=0.06)$. The prognostic value of systolic BPV for all-cause mortality was not affected by the region of study origin (North America-Europe vs. Asia), follow-up duration ( $\leq 2.5$ vs. $>2.5$ years), or variable type (BPV as a categorical vs. continuous variable) (Table 3 ; online suppl. Fig. 1-3).

When stratified by different metrics of systolic BPV, increased CV (HR: 1.17; 95\% CI: 1.03-1.31; $p=0.01$ ), SD (HR: $1.09 ; 95 \%$ CI: $1.03-1.16 ; p=0.003$ ), and ARIR (HR: 
a

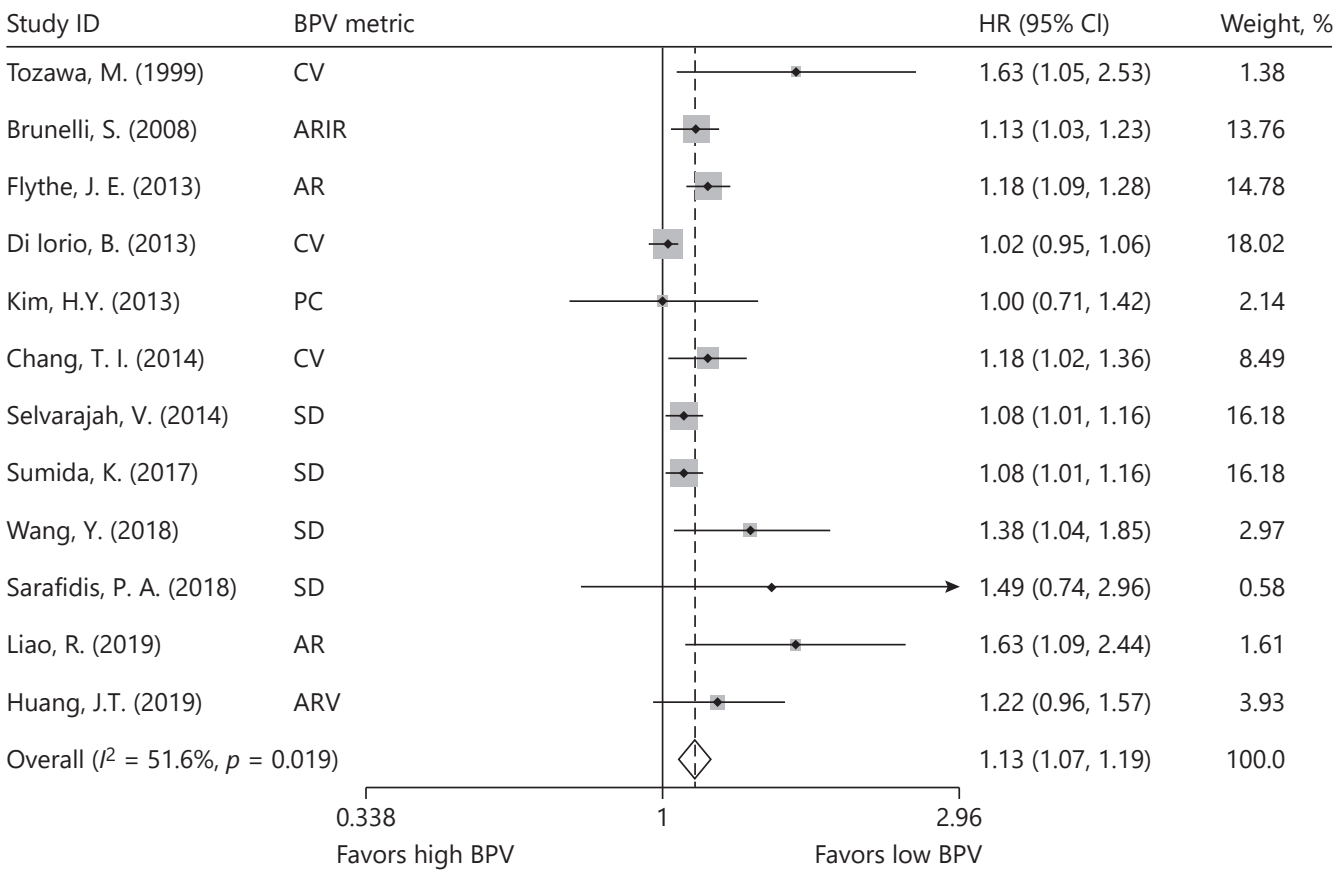

b

Study ID

$\mathrm{HR}(95 \% \mathrm{Cl})$

Weight, \%

Interdialytic BPV

Tozawa, M. (1999)

Brunelli, S. (2008)

Di lorio, B. (2013)

Kim, H.Y. (2013)

Chang, T. I. (2014)

Selvarajah, V. (2014)

Sumida, K. (2017)

Wang, Y. (2018)

Liao, R. (2019)

Subtotal $\left(R^{2}=52.7 \%, p=0.031\right)$

Intradialytic BPV

Flythe, J.E. (2013)

Kim, H.Y. (2013)

Liao, R. (2019)

Subtotal $\left(R^{2}=52.0 \%, p=0.125\right)$

Abulatory BPV

Sarafidis, P.A. (2018)

Huang, J.T. (2019)

Subtotal $\left(R^{2}=0.0 \%, p=0.598\right)$

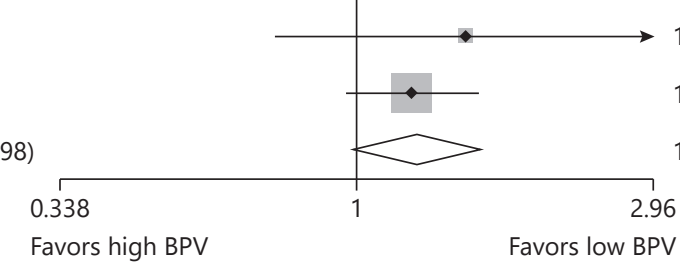

$1.63(1.05,2.53) \quad 1.66$

$1.13(1.03,1.23) \quad 17.04$

$1.02(0.95,1.06) \quad 22.51$

$1.00(0.71,1.42) \quad 2.58$

$1.18(1.02,1.36) \quad 10.40$

$1.08(1.01,1.16) \quad 20.13$

$1.08(1.01,1.16) \quad 20.13$

$1.38(1.04,1.85) \quad 3.59$

$1.63(1.09,2.44) \quad 1.94$

$1.11(1.05,1.17) \quad 100.00$

$1.18(1.09,1.28) \quad 54.80$

$0.99(0.83,1.18) \quad 34.18$

$0.93(0.62,1.39) \quad 11.02$

$1.08(0.93,1.26) \quad 100.00$

$1.49(0.74,2.96) \quad 11.03$

$1.22(0.96,1.57) \quad 88.97$

$1.25(0.99,1.57) \quad 100.00$

2

Favors high BPV

6
96

(Figure continued on next page.) 


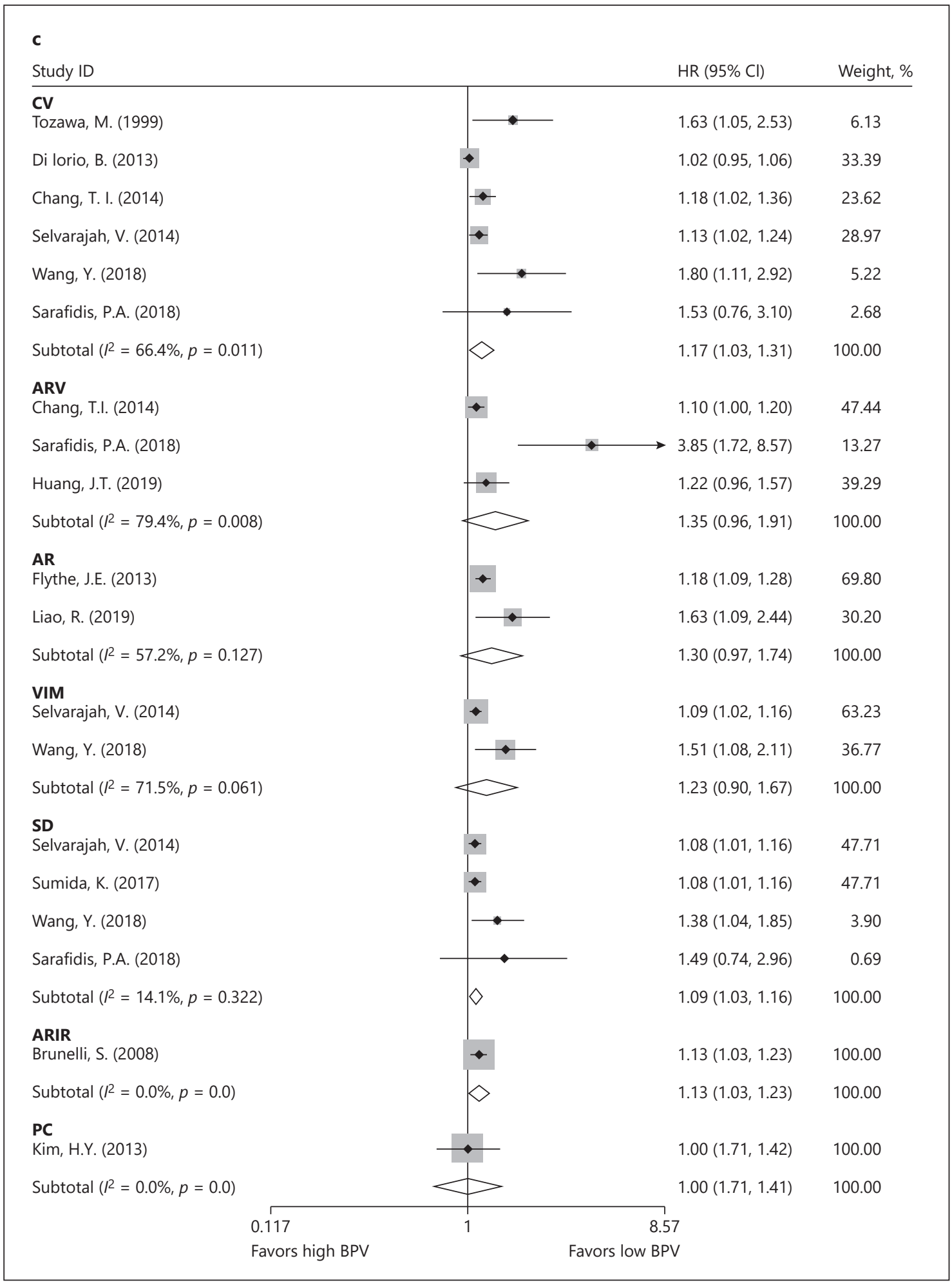

Fig. 2. a Meta-analysis of all-cause mortality in a random-effects model. b Meta-analysis of all-cause mortality by type of BPV in a random-effects model. c Meta-analysis of all-cause mortality by BPV metrics in a randomeffects model. AR, absolute residual; ARIR, average residual-intercept ratio; ARV, average real variability; BPV, blood pressure variability; CV, coefficient of variation; HR, hazard ratio; CI, confidence interval; PC, pressure change; SD, standard deviation; VIM, variation independent of mean. 


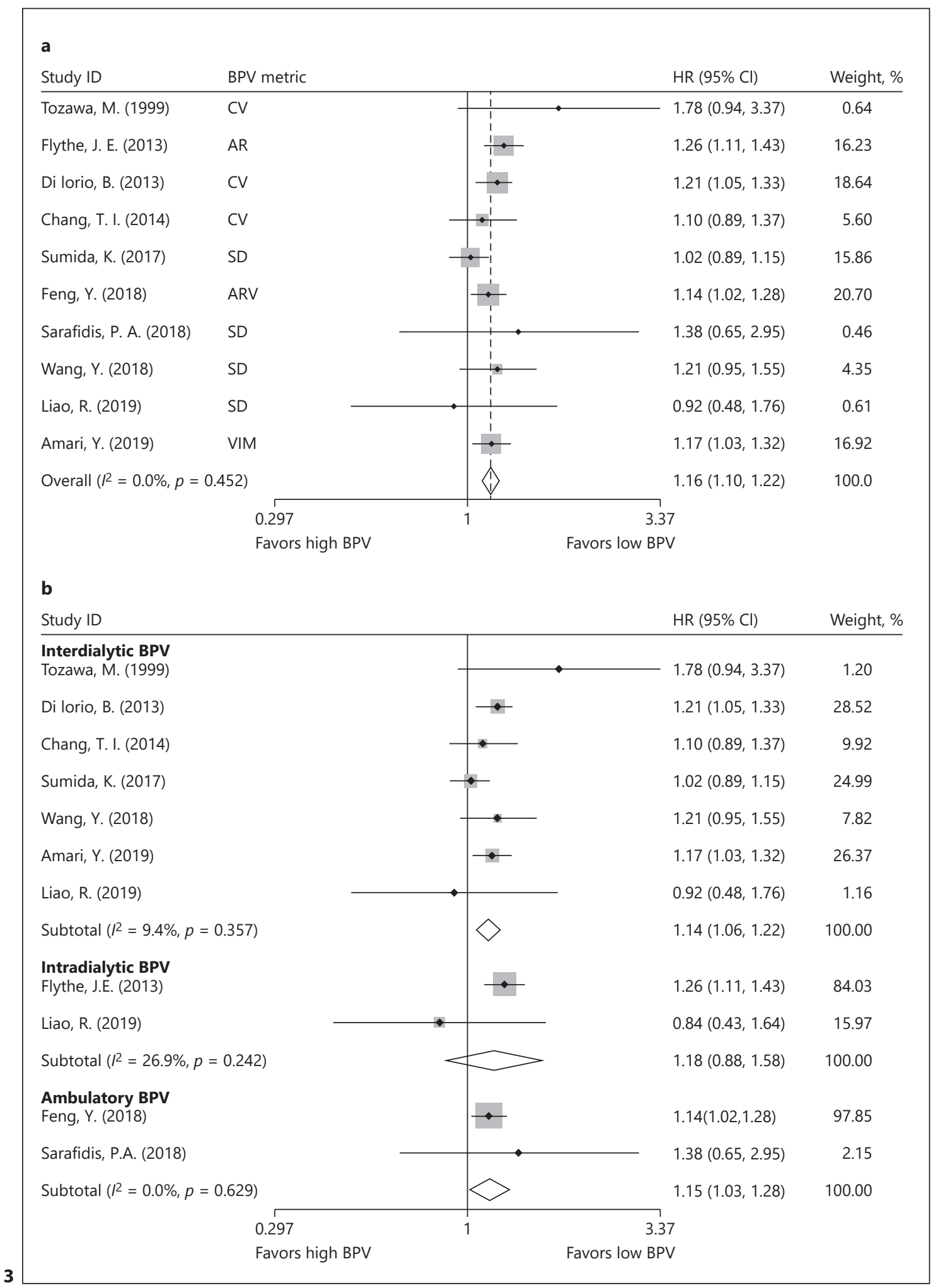

(Figure continued on next page.) 


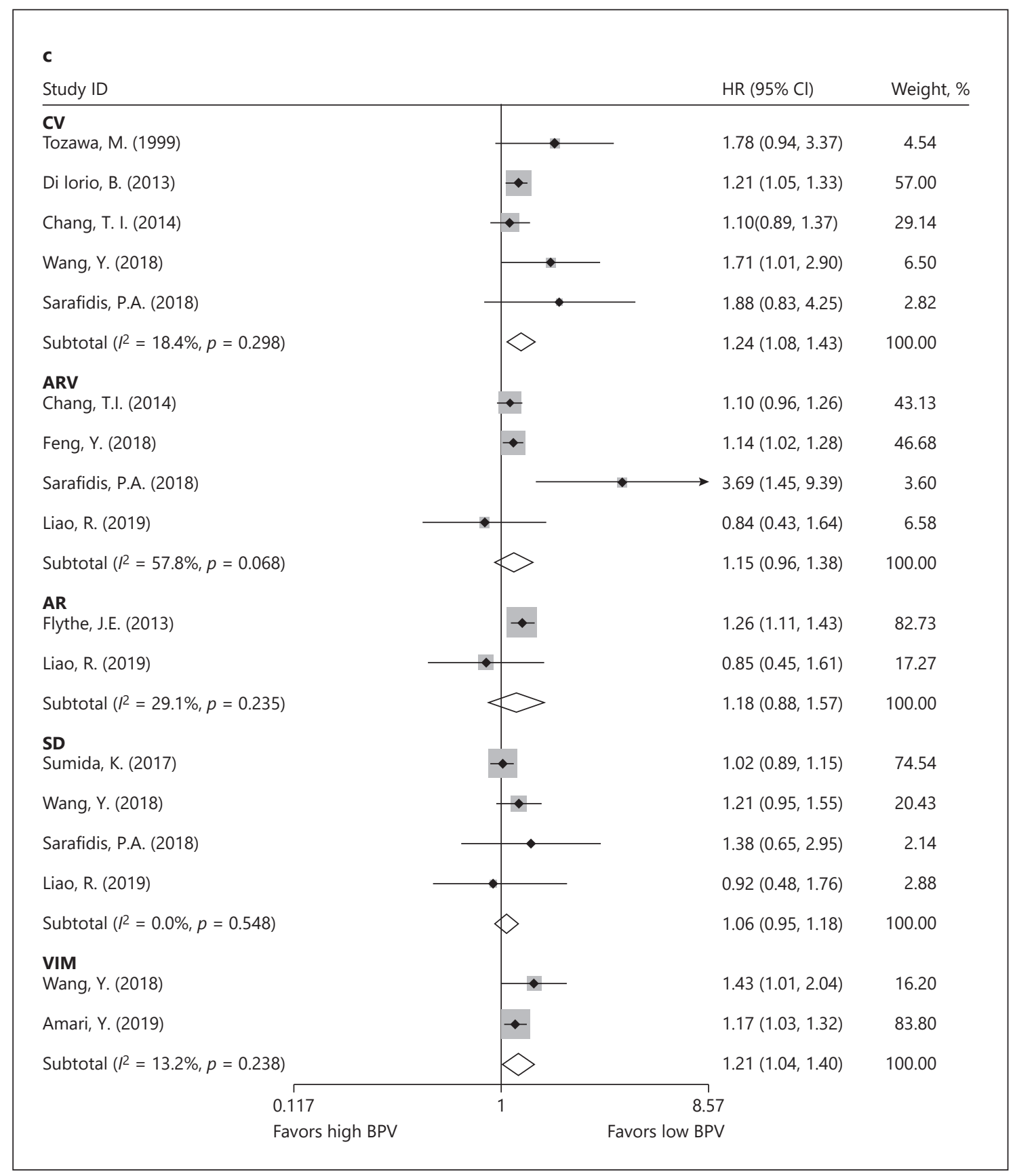

Fig. 3. a Meta-analysis of cardiovascular mortality in a random-effects model. b Meta-analysis of cardiovascular mortality by type of BPV in a random-effects model. c Meta-analysis of cardiovascular mortality by BPV metrics in a random-effects model. AR, absolute residual; ARV, average real variability; BPV, blood pressure variability; $\mathrm{CV}$, coefficient of variation; HR, hazard ratio; CI, confidence interval; PC, pressure change; SD, standard deviation; VIM, variation independent of mean.

1.13; 95\% CI: $1.03-1.23 ; p=0.007)$ demonstrated significant associations with all-cause mortality, whereas ARV (HR: 1.35; 95\% CI: 0.96-1.91; $p=0.08$ ), VIM (HR: 1.23; 95\% CI: 0.90-1.67; $p=0.19$ ), AR (HR: 1.30; 95\% CI: $0.97-$
1.74; $p=0.08$ ), and PC (HR: $1.00 ; 95 \%$ CI: $0.71-1.41 ; p=$ $1.00)$ did not demonstrate prognostic significance, possibly due to the limited number of eligible studies (Fig. 2c). 


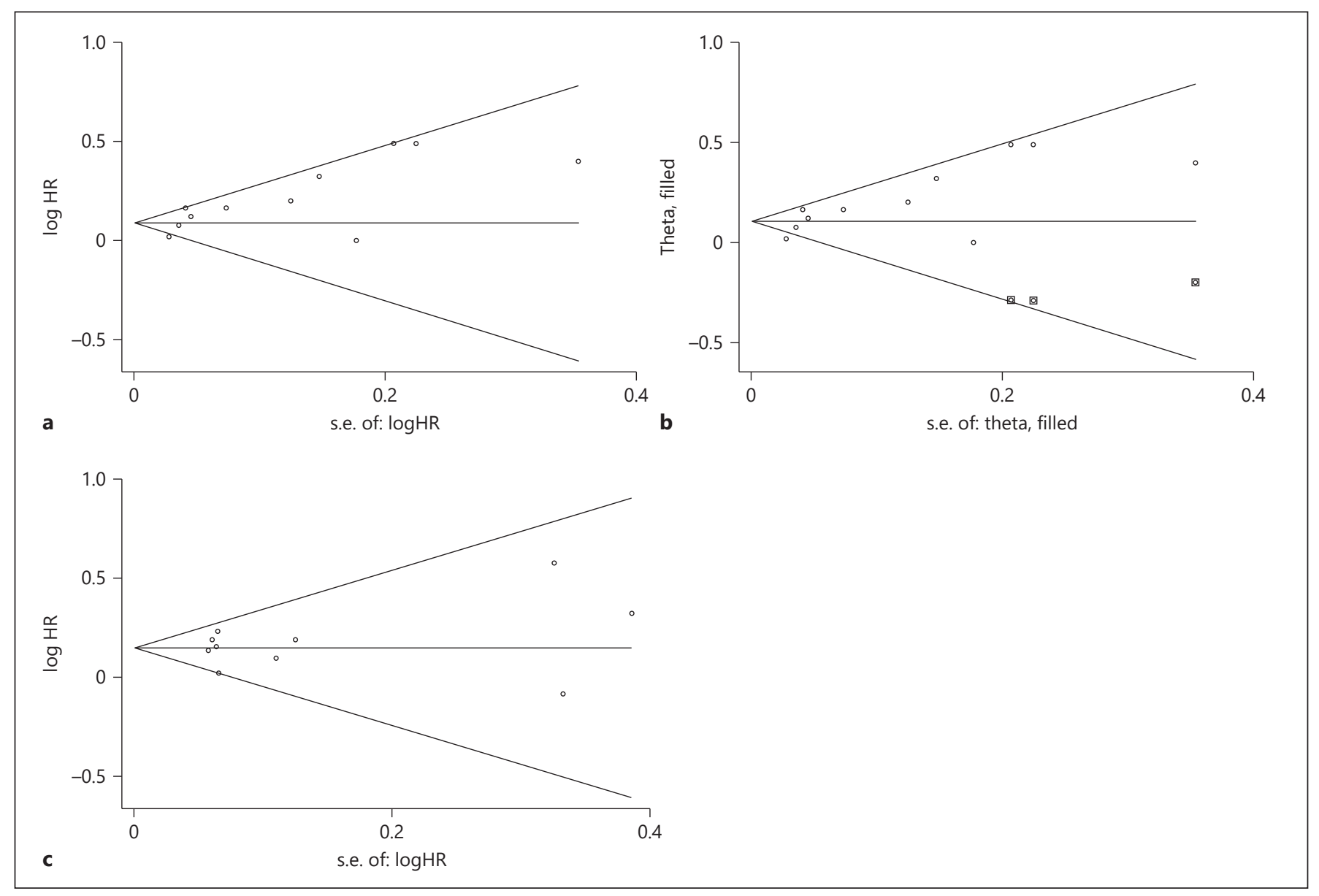

Fig. 4. a Begg's funnel plot for all-cause mortality. b Trim and fill method-adjusted Begg's funnel plot for all-cause mortality. c Begg's funnel plot for cardiovascular mortality.

\section{Diastolic BPV}

Five studies reported the prognostic value of diastolic BPV for all-cause mortality. Diastolic BPV was associated with all-cause mortality in patients receiving hemodialysis (HR: $1.09 ; 95 \% \mathrm{CI}: 1.01-1.17 ; p=0.02$ ) (online suppl. Fig. 4). Considering the limited number of studies included, stratified analysis was not performed for diastolic BPV on all-cause mortality.

\section{Cardiovascular Mortality}

Systolic BPV

A total of 10 studies involving 28,489 patients were included. In an overall analysis, we identified a significant association between systolic BPV and cardiovascular mortality in patients receiving hemodialysis (HR: 1.16; 95\% CI: $1.10-1.22 ; p<0.001)$. No significant betweenstudy heterogeneity was detected using the $\chi^{2}$ test and $I^{2}$ statistics $\left(I^{2}=0 \% ; p=0.45\right)$ (Fig. 3a). Interdialytic sys- tolic BPV (HR: 1.14; 95\% CI: $1.06-1.22 ; p<0.001$ ) and 44-h ambulatory systolic BPV (HR: 1.15; 95\% CI: $1.03-$ $1.28 ; p=0.015)$ were found to be related to cardiovascular mortality but intradialytic systolic BPV (HR: 1.18; 95\% CI: $088-1.58 ; p=0.266$ ) was not (Fig. 3b). In further analysis, the significant association between systolic BPV and cardiovascular mortality was not affected by the region of study origin (North America-Europe vs. Asia), follow-up duration ( $\leq 2.5$ vs. $>2.5$ years), or variable type (BPV as a categorical vs. continuous variable) (Table 3 ; online suppl. Fig. 5-7).

As can be seen in Figure 3c, stratified analysis by systolic BPV metrics indicated CV (HR: 1.24; 95\% CI: 1.08 $1.43 ; p=0.002$ ) and VIM (HR: $1.21 ; 95 \%$ CI: 1.04-1.40; $p=0.01)$ as predictors of cardiovascular death, whereas SD (HR: 1.06; 95\% CI: 0.95-1.18; $p=0.30$ ), ARV (HR: 1.15; 95\% CI: $0.96-1.38 ; p=0.14$ ), and AR (HR: 1.18 ; 95\% CI: $0.88-1.57 ; p=0.27)$ failed to reach a statistical significance. 
Table 3. Subgroup analysis for all-cause mortality and cardiovascular mortality

\begin{tabular}{llrrrrrrr}
\hline & & HR & $95 \%$ CI & $p$ value & & HR & 95\% CI & $p$ value \\
\hline All-cause mortality & & & & & & & & \\
$\quad$ Region & North America-Europe & 1.10 & $1.05-1.15$ & $<0.001$ & Asia & 1.31 & $1.11-1.54$ & 0.001 \\
$\quad$ Follow-up & Long & 1.24 & $1.04-1.47$ & 0.014 & Short & 1.12 & $1.07-1.16$ & $<0.001$ \\
$\quad$ Variable type & Continuous & 1.14 & $1.09-1.19$ & $<0.001$ & Categorical & 1.14 & $1.04-1.26$ & 0.008 \\
Cardiovascular mortality & & & & & & & & \\
$\quad$ Region & North America-Europe & 1.15 & $1.05-1.27$ & 0.003 & Asia & 1.16 & $1.08-1.26$ & $<0.001$ \\
$\quad$ Follow-up & Long & 1.20 & $1.10-1.29$ & $<0.001$ & Short & 1.13 & $1.03-1.24$ & 0.008 \\
$\quad$ Variable type & Continuous & 1.22 & $1.10-1.34$ & $<0.001$ & Categorical & 1.15 & $1.08-1.22$ & $<0.001$ \\
\hline
\end{tabular}

$\mathrm{HR}$, hazard ratio; CI, confidence interval.

Diastolic BPV

Only 2 studies reported the association between diastolic BPV and cardiovascular mortality. Diastolic BPV failed to predict cardiovascular death in patients receiving hemodialysis (HR: 0.86; 95\% CI: 0.52-1.42; $p=0.56$ ) (online suppl. Fig. 8). Similarly, stratified analysis was not performed for diastolic BPV on cardiovascular mortality due to the limited number of eligible studies.

\section{Composite Cardiovascular Events}

As only 2 studies described composite cardiovascular events, meta-analysis was not performed for this outcome. A prospective cohort study involving 227 patients receiving hemodialysis reported that fatal and nonfatal cardiovascular events combined were associated with increased 44-h ambulatory BPV (HR: 3.10; 95\% CI: 1.655.82; $p<0.001)$ in the crude analysis [10]. This finding was further supported by a retrospective cohort of 526 patients receiving hemodialysis focusing on intradialytic BPV in a fully adjusted model (HR: 1.31; 95\% CI: $1.04-$ $1.66 ; p=0.02)[25]$.

\section{Sensitivity Analysis and Publication Bias}

To assess the stability of the results, we performed a one-study-removed sensitivity analysis for systolic BPV on all-cause and cardiovascular mortality. Statistically similar results were obtained after individually omitting each study in both outcomes, indicating the stability of this meta-analysis (online suppl. Tables 4, 5). Publication bias was evaluated using Egger's test. For all-cause mortality, Egger's test demonstrated a statistically significant publication bias $(p=0.009)$. However, the results did not change substantially after adjustment with a nonparametric trim and fill method [26] (Fig. 4a, b; online suppl. Table 6). For cardiovascular mortality, no statistically significant publication bias was identified $(p=0.63)$ (Fig. 4c). The PRISMA checklist of this systematic review and meta-analysis is presented in online suppl. Table 7.

\section{Discussion}

This systematic review and meta-analysis of 14 cohort studies demonstrated that interdialytic systolic BPV was associated with an increased risk of both all-cause and cardiovascular mortality, whereas intradialytic systolic BPV failed to demonstrate such associations. The relationship between systolic BPV and prognosis was not affected by the region of study origin, follow-up duration, or variable type of BPV. Stratified analyses indicated that the CV of systolic blood pressure that predicted both allcause and cardiovascular mortality might be a BPV metric of clinical value in patients receiving hemodialysis. Diastolic BPV was found to be generally associated with all-cause mortality but failed to demonstrate a significant association with cardiovascular mortality in this metaanalysis.

The mechanism of altered BPV is complex. Behavioral, emotional, and postural factors could affect short-term BPV by influencing cardiovascular physiology [27], whereas arterial stiffness contributes to both short- [28] and long-term BPV [29]. In addition, the use of certain categories of antihypertensive drugs and adherence issues could result in BPV disorders [30, 31]. In addition to the aforementioned risk factors, patients receiving hemodialysis also have severe blood volume fluctuations during dialysis sessions. Compared with other populations, there 
is a significant elevation in BPV among the hemodialysis population [6]. One study also showed that BPV was increased on interdialytic day 2 , compared with day 1 , in patients receiving hemodialysis [32]. It is known that patients receiving hemodialysis demonstrate a poor prognosis because of an increased prevalence of CVDs [15], but whether BPV plays a role has been a topic of debate in the past decade.

Heterogeneity in the BPV metrics and measurement protocols across studies are some of the challenges in evaluating the predictive utility of BPV [33]. Among the 14 cohort studies included, a variety of BPV metrics, including CV, SD, ARV, VIM, AR, ARIR, and PC, were used. Past meta-analyses in the general population identified ARV as a useful approach to study the clinical value of BPV [4]; however, it did provide insights into whether certain BPV metrics should be preferred to others. To the best of our knowledge, this study is the first meta-analysis with a stratified analysis by BPV metric in the hemodialysis population. Although the failure of some metrics to reach statistical significance might be attributed to an insufficient number of eligible studies, there is evidence for the use of CV for BPV measurement. Furthermore, unlike blood pressure, BPV cannot be measured directly. Choosing a convenient and user-friendly BPV metric could further incentivize physicians to account for both blood pressure and BPV concurrently. $\mathrm{CV}$, which can be easily measured, can be a potential indicator of BPV in clinical settings.

Diastolic BPV has been reported to be associated with hemodialysis-related mortality [16], but this finding was not consistent in several other studies [10, 19-21]. Our analysis demonstrated that both systolic and diastolic BPV were significantly associated with all-cause mortality, whereas diastolic BPV failed to predict cardiovascular death. Interdialytic or intervisit BPV have been used by most studies to represent long-term BPV, whereas ambulatory and intradialytic BPV are typically classified as short-term BPV. In the general hypertensive population, intervisit BPV is confirmed by different meta-analyses as a risk factor of mortality and CVD with modest associations [1-3], whereas analyses of short-term BPV were hindered by limited data [3]. Similarly, in our meta-analysis on the hemodialysis population, we identified an association between interdialytic BPV and clinical outcomes. As different types of BPV signify different physiological and pathophysiological phenomena, more studies dedicated to 44-h ambulatory BPV and intradialytic BPV are needed to draw a solid conclusion regarding their predictive value in patients receiving hemodialysis.
According to the meta-analyses involving the general population [1-4], the HRs of BPV ranged from 1.14 to 1.20 for all-cause mortality and from 1.18 to 1.22 for cardiovascular mortality. It is interesting that in our study, BPV appeared to have a similar (if not less) impact on patients with uremia compared to the general hypertensive population. The underlying pathophysiology and clinical implications for these observations need to be further clarified. Nevertheless, considering the high incidence of cardiovascular events in the hemodialysis population, even a modest association with BPV is worth paying attention to.

Several limitations need to be considered while interpreting our findings. First, a variation in the methodological details (e.g., the different BPV types, metric types, time intervals, devices used for BPV measurements, and number of readings) could have affected the outcomes and added to the heterogeneity. Even in fully adjusted multivariate regressions, studies adjusted for different confounding factors could also have contributed to the heterogeneity. Although we analyzed the source of heterogeneity through meta-regression analyses and addressed the protocol variability by stratified analysis, notable heterogeneity continued to exist in several subgroups, which calls for further in-depth analysis. Second, the studies with large sample sizes were derived from the registry database or secondary analysis of clinical trials. In contrast, the studies dedicated to BPV were smaller observational cohorts. Because of the limited number and quality of studies, the results of some pooled-analyses (such as those of diastolic BPV) must be interpreted with caution. Third, our search was restricted to the selected literature databases. The unavailability of unreported outcomes in other studies could possibly result in a reporting bias. Last, the studies were conducted in North American, European, and East Asian countries. The applicability of our findings to other regions and other populations remains to be clarified.

In conclusion, interdialytic systolic BPV was associated with increased all-cause and cardiovascular mortality in the hemodialysis population. CV of systolic blood pressure was identified as a potentially promising metric of BPV in predicting all-cause and cardiovascular mortality. The clinical value of 44-h ambulatory systolic BPV, intradialytic systolic BPV, and the metrics of diastolic $\mathrm{BPV}$ warrants further investigations. More studies are required to understand whether BPV can be used as a therapeutic target in this patient population. 


\section{Statement of Ethics}

This study was performed in accordance with the Cochrane Handbook and PRISMA guidelines. The protocol for this review is registered with PROSPERO (CRD42019139215). The authors have no ethical conflicts to disclose. Informed consent and ethics approval were not required as this was a systematic review and meta-analysis.

\section{Conflict of Interest Statement}

The authors have no conflicts of interest to disclose.

\section{Funding Sources}

This study was funded by the National Natural Science Foundation of China (81700588) and the 1.3.5 Project for Disciplines of Excellence-Clinical Research Incubation Project, West China Hospital, Sichuan University (2020HXFH014). The funding sources had no role in the design or conduct of the study or the collection, management, analysis, or interpretation of the data.

\section{Author Contributions}

Conception and design: Yuliang Zhao, Ling Zhang, Tianlei Cui, and Ping Fu; literature search and selection: Yuliang Zhao, Letian Yang, and Shaobin Yu; data collection: Yuliang Zhao, Letian Yang, and Shaobin Yu; statistical analysis: Yuliang Zhao, Yi $\mathrm{Li}$, and Stephen Salerno; manuscript writing: Yuliang Zhao, Letian Yang, and Tianlei Cui; critical revision: Tianlei Cui, Ling Zhang, and Ping Fu; obtaining funding: Yuliang Zhao.

\section{References}

1 Wang J, Shi X, Ma C, Zheng H, Xiao J, Bian $\mathrm{H}$, et al. Visit-to-visit blood pressure variability is a risk factor for all-cause mortality and cardiovascular disease: a systematic review and meta-analysis. J Hypertens. 2017;35(1): $10-7$.

2 Diaz KM, Tanner RM, Falzon L, Levitan EB, Reynolds K, Shimbo D, et al. Visit-to-visit variability of blood pressure and cardiovascular disease and all-cause mortality: a systematic review and meta-analysis. Hypertension. 2014;64(5):965-82.

3 Stevens SL, Wood S, Koshiaris C, Law K, Glasziou P, Stevens RJ, et al. Blood pressure variability and cardiovascular disease: systematic review and meta-analysis. BMJ. 2016; 354:i4098.

4 Mena LJ, Felix VG, Melgarejo JD, Maestre GE. 24-hour blood pressure variability assessed by average real variability: a systematic review and meta-analysis. J Am Heart Assoc. 2017;6(10):e006895.

5 Hommos M, Schinstock C. Hypertension in the hemodialysis patient. Adv Exp Med Biol. 2017;956:327-40.

6 Rossignol P, Cridlig J, Lehert P, Kessler M, Zannad F. Visit-to-visit blood pressure variability is a strong predictor of cardiovascular events in hemodialysis: insights from FOSIDIAL. Hypertension. 2012;60(2):339-46.

7 Tang J, Yu S, Zhang Y, Xu Y. Clinical significance of blood pressure variability in chronic kidney disease and hemodialytic patients. Chin J Evid-Based Med. 2018;18:1018-21.

8 Tozawa M, Iseki K, Yoshi S, Fukiyama K. Blood pressure variability as an adverse prognostic risk factor in end-stage renal disease. Nephrol Dial Transplant. 1999;14(8):197681.

BPV and Prognosis in Hemodialysis Patients
9 Di Iorio B, Di Micco L, Torraca S, Sirico ML, Guastaferro P, Chiuchiolo L, et al. variability of blood pressure in dialysis patients: a new marker of cardiovascular risk. J Nephrol. 2013;26(1):173-82.

10 Sarafidis PA, Loutradis C, Karpetas A, Tzanis G, Bikos A, Raptis V, et al. The association of interdialytic blood pressure variability with cardiovascular events and all-cause mortality in haemodialysis patients nephrology, dialysis, transplantation: official publication of the European dialysis and transplant Association: European Renal Association. Nephrol Dial Transplant. 2019;34(3):515-23.

11 Chang TI, Flythe JE, Brunelli SM, Muntner P, Greene T, Cheung AK, et al. Visit-to-visit systolic blood pressure variability and outcomes in hemodialysis. J Hum Hypertens. 2014; 28(1):18-24.

12 Hayden JA, van der Windt DA, Cartwright JL, Côté P, Bombardier C. Assessing bias in studies of prognostic factors. Ann Intern Med. 2013;158(4):280-6.

13 Palmer SC, Hayen A, Macaskill P, Pellegrini F, Craig JC, Elder GJ, et al. Serum levels of phosphorus, parathyroid hormone, and calcium and risks of death and cardiovascular disease in individuals with chronic kidney disease: a systematic review and meta-analysis. JAMA. 2011;305(11):1119-27.

14 Mathew A, Devereaux PJ, O'Hare A, Tonelli M, Thiessen-Philbrook H, Nevis IF, et al. Chronic kidney disease and postoperative mortality: a systematic review and meta-analysis. Kidney Int. 2008;73(9):1069-81.

15 Amari Y, Morimoto S, Iida T, Yurugi T, Oyama Y, Aoyama N, et al. Characteristics of visit-to-visit blood pressure variability in hemodialysis patients. Hypertens Res. 2019;42(7): 1036-48.
16 Brunelli SM, Thadhani RI, Lynch KE, Ankers ED, Joffe MM, Boston R, et al. Association between long-term blood pressure variability and mortality among incident hemodialysis patients. Am J Kidney Dis. 2008;52(4):71626.

17 Feng Y, Li Z, Liu J, Sun F, Ma L, Shen Y, et al. Association of short-term blood pressure variability with cardiovascular mortality among incident hemodialysis patients. Ren Fail. 2018;40(1):259-64.

18 Flythe JE, Inrig JK, Shafi T, Chang TI, Cape K, Dinesh K, et al. Association of intradialytic blood pressure variability with increased allcause and cardiovascular mortality in patients treated with long-term hemodialysis. Am J Kidney Dis. 2013;61(6):966-74.

19 Kim HY, Kang YU, Kim CS, Choi JS, Bae EH, $\mathrm{Ma}$ SK, et al. Association of age and BP variability with long-term mortality in hemodialysis patients. Kidney Blood Press Res. 2013; 38(2-3):172-80.

20 Liao R, Li J, Lin L, Sun S, Wang L, Xiong Y, et al. The association between long- and intradialytic blood pressure variability with allcause mortality in hemodialysis patients. Blood Purif. 2019;48(1):43-50.

21 Selvarajah V, Pasea L, Ojha S, Wilkinson IB, Tomlinson LA. Pre-dialysis systolic blood pressure-variability is independently associated with all-cause mortality in incident haemodialysis patients. PloS One. 2014;9(1): e86514.

22 Sumida K, Molnar MZ, Potukuchi PK, Thomas F, Lu JL, Yamagata K, et al. Pre-end-stage renal disease visit-to-visit systolic blood pressure variability and post-end-stage renal disease mortality in incident dialysis patients. J Hypertens. 2017;35(9):1816-24. 
23 Wang Y, Qin Y, Fan X, Cai J, Ye W, Xia J, et al. Variability in predialysis systolic blood pressure and long-term outcomes in hemodialysis patients. Kidney Blood Press Res. 2018; 43(1):115-24.

24 Huang J-T, Cheng H-M, Yu W-C, Lin Y-P, Sung S-H, Chen C-H. Increased nighttime pulse pressure variability but not ambulatory blood pressure levels predicts 14-year allcause mortality in patients on hemodialysis. Hypertension. 2019;74(3):660-8.

25 Liao R, Li J, Xiong Y, Lin L, Wang L, Sun S, et al. Association of peridialysis blood pressure and its variability with cardiovascular events in hemodialysis patients. Kidney Blood Press Res. 2018;43(4):1352-62.

26 Duval S, Tweedie R. Trim and fill: a simple funnel-plot-based method of testing and adjusting for publication bias in meta-analysis. Biometrics. 2000;56(2):455-63.
27 Parati G, Ochoa JE, Lombardi C, Bilo G. Assessment and management of blood-pressure variability. Nat Rev Cardiol. 2013;10(3):14355.

28 Schillaci G, Bilo G, Pucci G, Laurent S, Macquin-Mavier I, Boutouyrie P, et al. Relationship between short-term blood pressure variability and large-artery stiffness in human hypertension: findings from 2 large databases. Hypertension. 2012;60(2):369-77.

29 Okada H, Fukui $M$, Tanaka $M$, Inada $S$, Mineoka Y, Nakanishi N, et al. Visit-to-visit variability in systolic blood pressure is correlated with diabetic nephropathy and atherosclerosis in patients with type 2 diabetes. Atherosclerosis. 2012;220(1):155-9.
30 Webb AJ, Fischer U, Mehta Z, Rothwell PM. Effects of antihypertensive-drug class on interindividual variation in blood pressure and risk of stroke: a systematic review and metaanalysis. Lancet. 2010;375(9718):906-15.

31 Muntner P, Levitan EB, Joyce C, Holt E, Mann D, Oparil S, et al. Association between antihypertensive medication adherence and visit-to-visit variability of blood pressure. J Clin Hypertens. 2013;15(2):112-7.

32 Karpetas A, Loutradis C, Bikos A, Tzanis G, Koutroumpas G, Lazaridis AA, et al. Blood pressure variability is increasing from the first to the second day of the interdialytic interval in hemodialysis patients. J Hypertens. 2017; 35(12):2517-26.

33 Parati G, Ochoa JE, Lombardi C, Bilo G. Blood pressure variability: assessment, predictive value, and potential as a therapeutic target. Curr Hypertens Rep. 2015;17(4):537. 